\title{
Boost Invariant Marginally Trapped Surfaces in Minkowski 4-Space
}

\author{
Stefan Haesen and Miguel Ortega*
}

October 30, 2018

\begin{abstract}
The extremal and partly marginally trapped surfaces in Minkowski 4-space, which are invariant under the group of boost isometries, are classified. Moreover, it is shown that there do not exist extremal surfaces of this kind with constant Gaussian curvature. A procedure is given in order to construct a partly marginally trapped surface by gluing two marginally trapped surfaces which are invariant under the group of boost isometries. As an application, a proper star-surface is constructed.
\end{abstract}

\section{Introduction}

In the study of the singularity theorems in general relativity, trapped surfaces play a fundamental role. These surfaces, introduced by Penrose [7], have the physical property that the two null congruences normal to the surface are both converging. From the mathematical point of view, the light converging condition means that the mean curvature vector, which measures the tension of the surface coming from the surrounding space, is a timelike vector everywhere on the surface. If the mean curvature vector is future- or past-pointing all over the surface, the trapped surface is accordingly called future- or past-trapped.

The existence of closed trapped surfaces has been investigated in several types of spacetimes. For example, in $[4,5]$ the formation of trapped surfaces in several cosmological is studied, while in [6] the non-existence of these closed surfaces is shown for strictly stationary spacetimes. In [3] it is shown that the existence of one trapped surface in a spherically symmetric spacetime is sufficient to ensure the formation of a black hole and the completeness of null infinity. In [8] a generalization of the concept of trapped surface is given for codimension 2 submanifolds of a Lorentzian space.

If the condition on the mean curvature vector is relaxed to be a causal vector on the surface, the surface is called nearly trapped and if the mean curvature vector is every lightlike or null, it is called marginally trapped. In the mathematics literature, these surfaces are called quasi-minimal and were studied in, e.g., [11]. Although for their use in the singularity theorems it is crucial that the marginally trapped surfaces are compact, in recent work this condition is often not assumed. For example, in $[1,2]$ marginally trapped surfaces are studied

*The first author is partially supported by the Research Foundation - Flanders project G.07.0432, and both authors are partially supported by the MEC Grant MTM2007-60731 and the Junta de Andalucía Grant P06-FQM-01951. 
in Lorentzian space forms and Robertson-Walker spaces with an extra assumption on the second fundamental form.

A complete classification of spacelike surfaces in a 4-dimensional Lorentzian spacetime, containing the above cases, was recently given in [9]. In the following we make reference to the notation introduced there.

In this text we consider spacelike surfaces in the Minkowski space $\mathbb{L}^{4}$ which are invariant under the following subgroup of direct, linear isometries of $\mathbb{L}^{4}$,

$$
\mathbf{G}=\left\{B_{\theta}=\left(\begin{array}{cccc}
\cosh (\theta) & \sinh (\theta) & 0 & 0 \\
\sinh (\theta) & \cosh (\theta) & 0 & 0 \\
0 & 0 & 1 & 0 \\
0 & 0 & 0 & 1
\end{array}\right): \theta \in \mathbb{R}\right\},
$$

well-known as boost isometries. We also recall that they preserve the natural timelike orientation of $\mathbb{L}^{4}$. We obtain the following results:

1. In Theorem 4.1, we classify all G-invariant partly marginally trapped surfaces in $\mathbb{L}^{4}$, including a rigidity result. The rest of this paper is a collection of corollaries of this theorem and some examples. In particular, we exhibit how to construct examples of marginally trapped surfaces which were not covered by the classification in [1]. In fact, the condition of positive relative nullity assumed there can be geometrically interpreted as saying that the intrinsic and extrinsic curvature of the surface are the same. Hence, only the surfaces of Corollary 6.3 are covered by the classification in [1].

2. In Corollary 4.1, we classify all G-invariant extremal surfaces in $\mathbb{L}^{4}$, showing that they are contained in a totally geodesic $\mathbb{L}^{3}$.

3. In Section 5 a general procedure is given to construct G-invariant partly marginally trapped surfaces by gluing two G-invariant marginally trapped surfaces. This method can be easily extended to a countable family of such surfaces. As an application, in Example 7.4, a null future-trapped surface is constructed, while in Example 7.5 a method is given to construct various $*$-surfaces in Minkowski space. Recall [9] for the definition of $*$-surfaces.

4. Since the Gaussian curvature $K$ is invariant along the orbits of the action of $\mathbf{G}$, the function $K$ only depends on one parameter. In this way, in Corollary 6.1, we show that it is possible to construct a $\mathbf{G}$-invariant spacelike surface with prescribed Gaussian curvature. In addition, the surface might be extremal or marginally trapped, according to a condition on the profile curve. Also, it is possible that the time-orientation of the mean vector field may vary from future to past (or viceversa), on different regions of the surface where it is marginally trapped. In such case, the boundary of two such regions must be of extremal points. In other words, the surface is an example of a $*$-surface. Moreover, we construct an example of a G-invariant marginally trapped surface with non-constant bounded Gaussian curvature.

5. In Corollary 6.2, we show the non-existence of G-invariant extremal surfaces in $\mathbb{L}^{4}$ with constant Gaussian curvature.

6. In Corollary 6.3, we describe the G-invariant flat marginally trapped surfaces in $\mathbb{L}^{4}$. An open problem remains to find the $\mathbf{G}$-invariant marginally trapped surfaces with constant 
Gaussian curvature. In Example 7.2, we give a family of surfaces with negative constant Gaussian curvature. In Example 7.3, an example of a surface with constant Gaussian curvature $K=1$ is given, but by using transcendental functions, since the desired primitives cannot be explicitly computed.

\section{Set up}

Let $\mathbb{L}^{4}$ be the 4 -dimensional Lorentz-Minkowski space with the flat metric given in local coordinates by

$$
g_{o}=-\mathrm{d} x_{1}^{2}+\mathrm{d} x_{2}^{2}+\mathrm{d} x_{3}^{2}+\mathrm{d} x_{4}^{2} .
$$

Given a connected immersed surface $S$ in $\mathbb{L}^{4}$, we call $g$ the restriction of the metric $g_{o}$ to $S$. Also, we assume that the metric $g$ is positive-definite, i. e., the surface is space-like. Let $\nabla$ be the Levi-Civita connection of $\left(\mathbb{L}^{4}, g_{o}\right)$. Given two smooth vector fields $X, Y$ tangent to $S$, we denote the normal component of $\nabla_{X} Y$ by $\mathbf{K}(X, Y)$, where

$$
\mathbf{K}: \mathfrak{X}(S) \times \mathfrak{X}(S) \longrightarrow \mathfrak{X}^{\perp}(S),
$$

which is usually called the shape tensor of $S$ in $\mathbb{L}^{4}$. Given a normal vector $\eta$, the shape operator $A_{\eta}$ associated with $\eta$ is the endomorphism of $T S$ given by $g_{o}(\mathbf{K}(X, Y), \eta)=g\left(A_{\eta} X, Y\right)$, for any tangent vectors $X, Y$ to $S$. The mean curvature vector $H$ is the trace of the shape tensor,

$$
H=\frac{1}{2} \operatorname{tr}_{g} \mathbf{K} \in \mathfrak{X}^{\perp}(S) .
$$

The component of $H$ along a given normal direction $\eta$ is called the expansion along $\eta$, $g(H, \eta)=\operatorname{tr}_{g}\left(A_{\eta}\right)$.

A surface $S$ in $\mathbb{L}^{4}$ is boost invariant if it is invariant by the group $\mathbf{G}$, i. e., if $B_{\theta}(S)=S$ for any $\theta \in \mathbb{R}$. Hence, the spacelike surface $S$ has to lie in one of the two $\mathbf{G}$-invariant regions $\mathcal{R}=\left\{\left(x_{1}, x_{2}, x_{3}, x_{4}\right) \in \mathbb{L}^{4}:\left|x_{1}\right|>\left|x_{2}\right|, x_{1}>0\right\}$, or the one with $x_{1}<0$, respectively. In the following we will always assume $S \subset \mathcal{R}$, since the other case is analogous. Since the set of fixed points of $\mathbf{G}$ is $\left\{\left(x_{1}, x_{2}, x_{3}, x_{4}\right) \in \mathbb{L}^{4}: x_{1}=x_{2}=0\right\}$, we can introduce a parametrization $X(s, \theta)$ on a dense open subset $\Sigma_{\alpha}$ of $S$ as follows,

$$
\Sigma_{\alpha}=\left\{X(s, \theta)=\alpha(s) \cdot B_{\theta}: s \in I, \theta \in \mathbb{R}\right\},
$$

where

$$
\alpha: I \subset \mathbb{R} \longrightarrow \mathcal{P}=\left\{\left(x_{1}, x_{2}, x_{3}, x_{4}\right) \in \mathbb{L}^{4}: x_{2}=0, x_{1}>0\right\}, \alpha(s)=\left(\alpha_{1}(s), 0, \alpha_{3}(s), \alpha_{4}(s)\right) .
$$

Also, since the surface $\Sigma_{\alpha}$ has to be space-like (also called Riemannian), the curve $\alpha$ should be space-like, i. e.,

$$
-\left(\alpha_{1}^{\prime}\right)^{2}+\left(\alpha_{3}^{\prime}\right)^{2}+\left(\alpha_{4}^{\prime}\right)^{2}>0 .
$$

We consider a local orthonormal basis $\left\{\eta_{1}, \eta_{2}\right\}$ of the normal bundle of $\Sigma_{\alpha}$ in $\mathbb{L}^{4}$, where $\eta_{1}$ is future-pointing time-like and $\eta_{2}$ is space-like. If we denote by $A_{i}$ the shape operator associated with $\eta_{i}, i=1,2$, the shape tensor can be written as

$$
\mathbf{K}(X, Y)=-g\left(A_{1} X, Y\right) \eta_{1}+g\left(A_{2} X, Y\right) \eta_{2},
$$


for any tangent vector fields $X, Y$ to $\Sigma_{\alpha}$. From the classical theory of surfaces (see e.g. [10]), with the notation

$$
\begin{gathered}
E=g\left(X_{s}, X_{s}\right), \quad F=g\left(X_{s}, X_{\theta}\right), G=g\left(X_{\theta}, X_{\theta}\right), \\
e_{i}=g_{o}\left(X_{s s}, \eta_{i}\right), f_{i}=g_{o}\left(X_{s \theta}, \eta_{i}\right), g_{i}=g_{o}\left(X_{\theta \theta}, \eta_{i}\right), \\
h_{i}=\operatorname{tr}_{g}\left(A_{i}\right),
\end{gathered}
$$

we obtain

$$
h_{i}=\frac{e_{i} G-2 f_{i} F+g_{i} E}{E G-F^{2}}, i=1,2 .
$$

Also, another useful local basis $\{\mathbf{l}, \mathbf{k}\}$ of the normal bundle of $\Sigma_{\alpha}$ in $\mathbb{L}^{4}$ can be chosen such that they are null, future-pointing and satisfying the normalization condition $g_{o}(\mathbf{k}, \mathbf{l})=-1$. The basis can be changed by choosing a positive function defined on $S, \sigma$, in the following way,

$$
\mathbf{l} \longrightarrow \mathbf{l}^{\prime}=\sigma \mathbf{l}, \quad \mathbf{k} \longrightarrow \mathbf{k}^{\prime}=\sigma^{-1} \mathbf{k}
$$

In this note, we choose

$$
\mathbf{l}=\frac{1}{\sqrt{2}}\left(\eta_{1}+\eta_{2}\right), \quad \mathbf{k}=\frac{1}{\sqrt{2}}\left(\eta_{1}-\eta_{2}\right) .
$$

Now, the mean curvature vector field $H$ of $\Sigma_{\alpha}$ becomes

$$
H=\frac{1}{2}\left(-h_{1} \eta_{1}+h_{2} \eta_{2}\right)=-\frac{\sqrt{2}}{4}\left(h_{1}-h_{2}\right) \mathbf{l}-\frac{\sqrt{2}}{4}\left(h_{1}+h_{2}\right) \mathbf{k} .
$$

In particular, the expansions along $\mathbf{l}$ and $\mathbf{k}$ are given by

$$
\Theta_{\mathbf{l}}=\frac{\sqrt{2}}{4}\left(h_{1}+h_{2}\right), \quad \Theta_{\mathbf{k}}=\frac{\sqrt{2}}{4}\left(h_{1}-h_{2}\right) .
$$

We recall that a surface $S$ in $\mathbb{L}^{4}$ is called trapped if its mean curvature vector is everywhere time-like. If the mean curvature vector is always light-like and non-zero in at least one point, the surface is called marginally trapped. In case the mean curvature vector is null on some region of the surface and vanishes on another, the surface is called partly marginally trapped.

Besides the extrinsic mean curvature $H$, the intrinsic Gaussian curvature $K$ of the surface can be expressed in terms of the coefficients of the first and second fundamental forms as (see e.g. [10]),

$$
K=\frac{-\operatorname{det}\left(A_{1}\right)+\operatorname{det}\left(A_{2}\right)}{\operatorname{det}(g)}=\frac{-e_{1} g_{1}+e_{2} g_{2}+f_{1}^{2}-f_{2}^{2}}{E G-F^{2}}
$$

\section{$3 \quad$ Boost Invariant Surfaces}

We now consider a curve $\alpha: I \subset \mathbb{R} \rightarrow \mathcal{P}$ of the form

$$
\alpha(s)=\left(\alpha_{1}(s), 0, \alpha_{3}(s), \alpha_{4}(s)\right) .
$$

The parametrization of $\Sigma_{\alpha}$, which is invariant with respect to the rotation group $\mathbf{G}$, can be written down as

$$
X(s, \theta)=\left(\alpha_{1}(s) \cosh (\theta), \alpha_{1}(s) \sinh (\theta), \alpha_{3}(s), \alpha_{4}(s)\right), s \in I, \theta \in \mathbb{R} .
$$


We assume that the derivative of the curve $\alpha$ has constant length, so that $g_{o}\left(\alpha^{\prime}(s), \alpha^{\prime}(s)\right)=$ $c^{2}>0$ (for a suitable constant $c \in \mathbb{R}$ ). The derivatives of $X$ are

$$
X_{s}=\left(\alpha_{1}^{\prime} \cosh (\theta), \alpha_{1}^{\prime} \sinh (\theta), \alpha_{3}^{\prime}, \alpha_{4}^{\prime}\right), \quad X_{\theta}=\left(\alpha_{1} \sinh (\theta), \alpha_{1} \cosh (\theta), 0,0\right),
$$

and the Riemannian metric of the surface reads

$$
g=c^{2} \mathrm{~d} s^{2}+\alpha_{1}(s)^{2} \mathrm{~d} \theta^{2} .
$$

A globally defined orthonormal tangent frame on $\Sigma_{\alpha}$ is

$$
u_{1}=\frac{X_{s}}{c}, \quad u_{2}=\frac{X_{\theta}}{\alpha_{1}}
$$

and a globally defined orthonormal basis of the normal bundle of $\Sigma_{\alpha}$ is given by

$$
\begin{aligned}
\eta_{1} & =\frac{1}{c \sqrt{c^{2}+\left(\alpha_{1}^{\prime}\right)^{2}}}\left(\cosh (\theta)\left(c^{2}+\left(\alpha_{1}^{\prime}\right)^{2}\right), \sinh (\theta)\left(c^{2}+\left(\alpha_{1}^{\prime}\right)^{2}\right), \alpha_{1}^{\prime} \alpha_{3}^{\prime}, \alpha_{1}^{\prime} \alpha_{4}^{\prime}\right), \\
\eta_{2} & =\frac{1}{\sqrt{c^{2}+\left(\alpha_{1}^{\prime}\right)^{2}}}\left(0,0,-\alpha_{4}^{\prime}, \alpha_{3}^{\prime}\right),
\end{aligned}
$$

with $\eta_{1}$ future-pointing time-like and $\eta_{2}$ space-like. Moreover, the associated null basis is

$$
\begin{aligned}
\mathbf{l} & =\frac{1}{c \sqrt{2} \sqrt{c^{2}+\left(\alpha_{1}^{\prime}\right)^{2}}}\left(\cosh (\theta)\left(c^{2}+\left(\alpha_{1}^{\prime}\right)^{2}\right), \sinh (\theta)\left(c^{2}+\left(\alpha_{1}^{\prime}\right)^{2}\right), \alpha_{1}^{\prime} \alpha_{3}^{\prime}-c \alpha_{4}^{\prime}, \alpha_{1}^{\prime} \alpha_{4}^{\prime}+c \alpha_{3}^{\prime}\right) \\
\mathbf{k} & =\frac{1}{c \sqrt{2} \sqrt{c^{2}+\left(\alpha_{1}^{\prime}\right)^{2}}}\left(\cosh (\theta)\left(c^{2}+\left(\alpha_{1}^{\prime}\right)^{2}\right), \sinh (\theta)\left(c^{2}+\left(\alpha_{1}^{\prime}\right)^{2}\right), \alpha_{1}^{\prime} \alpha_{3}^{\prime}+c \alpha_{4}^{\prime}, \alpha_{1}^{\prime} \alpha_{4}^{\prime}-c \alpha_{3}^{\prime}\right) .
\end{aligned}
$$

A straightforward computation shows that

$$
\begin{gathered}
e_{1}=\frac{-c \alpha_{1}^{\prime \prime}}{\sqrt{c^{2}+\left(\alpha_{1}^{\prime}\right)^{2}}}, \quad f_{1}=0, \quad g_{1}=\frac{-\alpha_{1}}{c} \sqrt{c^{2}+\left(\alpha_{1}^{\prime}\right)^{2}}, \\
e_{2}=\frac{-\alpha_{4}^{\prime} \alpha_{3}^{\prime \prime}+\alpha_{4}^{\prime \prime} \alpha_{3}^{\prime}}{\sqrt{c^{2}+\left(\alpha_{1}^{\prime}\right)^{2}}}, \quad f_{2}=g_{2}=0, \\
h_{1}=-\frac{c^{2}+\left(\alpha_{1}^{\prime}\right)^{2}+\alpha_{1} \alpha_{1}^{\prime \prime}}{c \alpha_{1} \sqrt{c^{2}+\left(\alpha_{1}^{\prime}\right)^{2}}}, \quad h_{2}=\frac{-\alpha_{4}^{\prime} \alpha_{3}^{\prime \prime}+\alpha_{3}^{\prime} \alpha_{4}^{\prime \prime}}{c^{2} \sqrt{c^{2}+\left(\alpha_{1}^{\prime}\right)^{2}}} .
\end{gathered}
$$

Finally, the Gaussian curvature of a G-invariant surface $\Sigma_{\alpha}$ is

$$
K=-\frac{\alpha_{1}^{\prime \prime}}{c^{2} \alpha_{1}}
$$

Note that the surface $\Sigma_{\alpha}$ always lies on the diagonal of Table 3 in [9]. In the following we are interested in the particular cases that the surface is marginally trapped. 


\section{Main Result}

We note that given a smooth function $\rho: I \longrightarrow \mathbb{R}$, sometimes it is useful to modify it in such a way that it changes its sign on one (or more) connected component of $\{s \in I: \rho(s) \neq 0\}$, but it is still smooth after the modification. We define the set of points $Z_{\rho}=\{s \in I: \rho(s)=0\}$. Assume that there is a connected component $C$ of the interior points of $Z_{\rho}$, and a boundary point $s_{0} \in \partial C$. By the continuity of $\rho$, there is a real number $\delta>0$ such that (i) for any other connected component $\tilde{C}$ of the interior of $Z_{\rho}$, then $] s_{0}-\delta, s_{0}+\delta[\cap \tilde{C}=\emptyset$; and (ii) the set ]$s_{0}-\delta, s_{0}+\delta[\cap C$ is connected. Next, we can define a function $\varepsilon: C \cup] s_{0}-\delta, s_{0}+\delta[\longrightarrow\{-1,0,1\}$ such that (i) if $s \in C$, then $\varepsilon(s)=0$; (ii) $\varepsilon$ is constant $( \pm 1)$ on $] s_{0}-\delta, s_{0}+\delta[\backslash C$; and (iii) $\varepsilon \rho$ is smooth on $C \cup] s_{0}-\delta, s_{0}+\delta[$. We should point out that there might be more elements of $Z_{\rho}$ in $] s_{0}-\delta, s_{0}+\delta[$, but there smoothness of $\rho$ yields the choice of $\varepsilon$ as before. The choice of $\varepsilon$ can be extended to the whole domain of $\rho$ in such a way that $\varepsilon \rho$ is smooth on $I$. This way, we have the following lemma.

Lemma 4.1 Sign Choice. Given a smooth function $\rho: I \longrightarrow \mathbb{R}$, there exists many (maybe only constant) functions $\varepsilon: I \longrightarrow\{-1,0,1\} \subset \mathbb{R}$ such that $\varepsilon \rho$ is smooth on $I$, and if $s \in I$ such that $\rho(s) \neq 0$, then $\varepsilon(s)= \pm 1$.

Usually, the simplest choice consists of setting $\varepsilon$ as a constant on the whole domain of $\rho$. Different choices will be useful in Section 5 .

Theorem 4.1 Given a constant $c>0$ and a smooth function $\alpha_{1}>0$, we define the function

$$
\rho=-\frac{c^{2}+\left(\alpha_{1}^{\prime}\right)^{2}+\alpha_{1} \alpha_{1}^{\prime \prime}}{\alpha_{1}}
$$

We choose a function $\varepsilon$ as in Lemma 4.1, such that $\varepsilon \rho$ is smooth. Next, we define the following functions

$$
\xi=\int \frac{\varepsilon c \rho}{c^{2}+\left(\alpha_{1}^{\prime}\right)^{2}} \mathrm{~d} s, \quad \alpha_{3}=\int \sqrt{c^{2}+\left(\alpha_{1}^{\prime}\right)^{2}} \cos (\xi) \mathrm{d} s, \text { and } \alpha_{4}=\int \sqrt{c^{2}+\left(\alpha_{1}^{\prime}\right)^{2}} \sin (\xi) \mathrm{d} s .
$$

Then, the space-like curve $\alpha(s)=\left(\alpha_{1}(s), 0, \alpha_{3}(s), \alpha_{4}(s)\right)$ is a profile curve of a $\mathbf{G}$-invariant surface $\Sigma_{\alpha}$ with

$$
H=\frac{\rho}{2 c \sqrt{c^{2}+\left(\alpha_{1}^{\prime}\right)^{2}}}\left(-\eta_{1}+\varepsilon \eta_{2}\right), \quad g_{o}\left(\alpha^{\prime}, \alpha^{\prime}\right)=c^{2} .
$$

In addition,

1. The surface $\Sigma_{\alpha}$ is marginally trapped on the points where $\rho \neq 0$. Moreover, $H$ is futurepointing if, and only if, $\rho<0$.

2. The surface $\Sigma_{\alpha}$ is extremal on the points where $\rho=0$.

3. Given any other curve $\beta=\left(\beta_{1}, 0, \beta_{3}, \beta_{4}\right)$ such that $g_{o}\left(\beta^{\prime}, \beta^{\prime}\right)=c^{2}$ and $\beta_{1}=\alpha_{1}$, for each connected component $C$ of $\left\{s \in I: \rho_{\alpha}(s) \neq 0\right\}$, there exists an affine isometry $F$ of $\mathbb{L}^{4}$ such that $F\left(\Sigma_{\left.\alpha\right|_{C}}\right)=\Sigma_{\left.\beta\right|_{C}}$.

Conversely, any $\mathbf{G}$-invariant surface which is partly marginally trapped, admits a dense open subset of the form $\Sigma_{\alpha}$, with $\alpha: I \longrightarrow \mathcal{P}, g_{o}\left(\alpha^{\prime}, \alpha^{\prime}\right)=c^{2}$, such that $\Sigma_{\alpha}$ can be constructed as above. 
Proof: We first show the sufficient condition. Let $\alpha$ be a space-like curve satisfying $g_{o}\left(\alpha^{\prime}, \alpha^{\prime}\right)=$ $c^{2}>0$, with $c \in \mathbb{R}$ a constant. We can rewrite this expression as

$$
\alpha_{3}^{\prime}=\sqrt{c^{2}+\left(\alpha_{1}^{\prime}\right)^{2}} \cos (\xi), \quad \alpha_{4}^{\prime}=\sqrt{c^{2}+\left(\alpha_{1}^{\prime}\right)^{2}} \sin (\xi),
$$

for a suitable smooth angle function $\xi$. In this way, up to integration constants, we can recover $\alpha_{3}$ and $\alpha_{4}$ from the data $\xi$ and $\alpha_{1}$. Next, we assume that $\alpha$ is the profile curve of a G-invariant partly marginally trapped surface. This means $\|H\|^{2}=0$. First, we consider the case $H \neq 0$. On a small enough open subset of the domain of $\alpha$, there holds that $h_{1}=\varepsilon h_{2}$, with $\varepsilon= \pm 1$. Thus, by Lemma 4.1 , there exists a function $\varepsilon$ taking the values $\{-1,0,1\}$ such that $h_{1}=\varepsilon h_{2}$. Bearing in mind (11), we see that this is equivalent to

$$
\rho=-\frac{c^{2}+\left(\alpha_{1}^{\prime}\right)^{2}+\alpha_{1} \alpha_{1}^{\prime \prime}}{\alpha_{1}}=\varepsilon \frac{-\alpha_{4}^{\prime} \alpha_{3}^{\prime \prime}+\alpha_{3}^{\prime} \alpha_{4}^{\prime \prime}}{c},
$$

where $\rho$ is a smooth function. We should point out that when $H=0$, then $h_{1}=h_{2}=0$, so that we can choose $\rho=0$ (and $\varepsilon=0$ ) in such points. Next, by inserting (13) in (14), we obtain

$$
\xi^{\prime}=\frac{\varepsilon c \rho}{c^{2}+\left(\alpha_{1}^{\prime}\right)^{2}}
$$

This expression allows one to recover $\xi$ from $\rho$ and $\alpha_{1}$, up to an integration constant. Now, by (11) and (14), and recalling $\rho=h_{1} c \sqrt{c^{2}+\left(\alpha_{1}^{\prime}\right)^{2}}=\varepsilon h_{2} c \sqrt{c^{2}+\left(\alpha_{1}^{\prime}\right)^{2}}$, it readily follows that

$$
H=\frac{\rho}{2 c \sqrt{c^{2}+\left(\alpha_{1}^{\prime}\right)^{2}}}\left(-\eta_{1}+\varepsilon \eta_{2}\right) .
$$

From this expression, since $\eta_{1}$ is future-pointing, $H$ is future-pointing if, and only if, $\rho<0$. It only remains to check the rigidity condition of item 3. Indeed, if $\alpha_{1}=\beta_{1}$ and $g_{o}\left(\beta^{\prime}, \beta^{\prime}\right)=$ $c^{2}$, both functions $\alpha_{1}$ and $\beta_{1}$ determine the very same function $\rho$. Let $C$ be a connected component of $\left\{s \in I: \rho_{\alpha}(s) \neq 0\right\}$, so we work on $C$. Next, if $\xi_{\alpha}$ and $\xi_{\beta}$ are the corresponding angle functions, then $\xi_{\alpha}^{\prime}=\delta \xi_{\beta}^{\prime}$, for a suitable constant $\delta= \pm 1$, which depends on the functions $\varepsilon$ associated with $\alpha$ and $\beta$. This way, $\xi_{\alpha}=\delta \xi_{\beta}+\xi_{0}$, where $\xi_{0}$ is just an integration constant. An elementary computation shows

$$
\left(\beta_{3}^{\prime}, \beta_{4}^{\prime}\right)=\left(\alpha_{3}^{\prime}, \alpha_{4}^{\prime}\right)\left(\begin{array}{ll}
1 & 0 \\
0 & \delta
\end{array}\right)\left(\begin{array}{cc}
\cos \left(\xi_{0}\right) & \sin \left(\xi_{0}\right) \\
-\sin \left(\xi_{0}\right) & \cos \left(\xi_{0}\right)
\end{array}\right) .
$$

Next, if the integration constants are $\alpha_{3}^{0}$ and $\alpha_{4}^{0}$, we call $v=\left(0,0, \alpha_{3}^{0}, \alpha_{4}^{0}\right)$. It is clear that the affine isometry $F: \mathbb{L}^{4} \longrightarrow \mathbb{L}^{4}$,

$$
F\left(x_{1}, x_{2}, x_{3}, x_{4}\right)=\left(x_{1}, x_{2}, x_{3}, x_{4}\right)\left(\begin{array}{cccc}
1 & 0 & 0 & 0 \\
0 & 1 & 0 & 0 \\
0 & 0 & 1 & 0 \\
0 & 0 & 0 & \delta
\end{array}\right)\left(\begin{array}{cccc}
1 & 0 & 0 & 0 \\
0 & 1 & 0 & 0 \\
0 & 0 & \cos \left(\xi_{0}\right) & \sin \left(\xi_{0}\right) \\
0 & 0 & -\sin \left(\xi_{0}\right) & \cos \left(\xi_{0}\right)
\end{array}\right)+v
$$

satisfies $\left.F \circ \alpha\right|_{C}=\left.\beta\right|_{C}$ and thus $F\left(\Sigma_{\left.\alpha\right|_{C}}\right)=\Sigma_{\left.\beta\right|_{C}}$.

Finally, the converse is a straightforward computation from equations (13), (14) and (15). 
Corollary 4.1 Let $\Sigma_{\alpha}$ be a $\mathbf{G}$-invariant extremal surface. Then, a unit profile curve is given by

$$
\alpha(s)=\left(f(s), 0, \cos \left(\xi_{0}\right) \sqrt{a_{1}} \arctan \left(\frac{s+a_{2}}{f(s)}\right), \sin \left(\xi_{0}\right) \sqrt{a_{1}} \arctan \left(\frac{s+a_{2}}{f(s)}\right)\right),
$$

where $f(s)=\sqrt{a_{1}-\left(s+a_{2}\right)^{2}}$, and $a_{1}, a_{2}, \xi_{0} \in \mathbb{R}, a_{1}>0$, being integration constants. In particular, $\Sigma_{\alpha}$ is immersed in a totally geodesic Lorentzian 3-plane.

Proof: If we assume $H=0$, we have $h_{1}=h_{2}=0$. By (11), with $c=1$, we obtain

$$
1+\left(\alpha_{1}^{\prime}\right)^{2}+\alpha_{1} \alpha_{1}^{\prime \prime}=0 \text { and }-\alpha_{4}^{\prime} \alpha_{3}^{\prime \prime}+\alpha_{3}^{\prime} \alpha_{4}^{\prime \prime}=0 .
$$

Now, the general solution of (16) is $\alpha_{1}(s)=\varepsilon \sqrt{a_{1}-\left(s+a_{2}\right)^{2}}$, where $a_{1}, a_{2} \in \mathbb{R}$ are integration constants with $a_{1}>0$ and $\varepsilon= \pm 1$. However, since we are always assuming $\alpha_{1}$ to be positive, the case $\varepsilon=-1$ cannot occur. Next, we make use of Theorem 4.1. This way, $\rho=0$, which readily implies $\xi(s)=\xi_{0}$ for a certain constant $\xi_{0} \in \mathbb{R}$. A straightforward computation gives the expressions of $\alpha_{3}$ and $\alpha_{4}$. In addition, the second equation of (16) implies that the shape operator $A_{2}=0$. Finally, we show that the surface is contained in a totally geodesic $\mathbb{L}^{3}$. To do so, we observe that the curve $\alpha$ is contained in the Lorentzian 2-plane $\left\{\left(x_{1}, x_{2}, x_{3}, x_{4}\right) \in \mathbb{L}^{4}: x_{2}=0, \sin \left(\xi_{0}\right) x_{3}=\cos \left(\xi_{0}\right) x_{4}\right\}$ and that the $\mathbf{G}$-orbits are orthogonal to this plane.

\section{Gluing G-Invariant Marginally Trapped Surfaces}

The aim of this section is to give a general method to construct a $\mathbf{G}$-invariant partly marginally trapped surface from two G-invariant marginally trapped surfaces.

We recall that the following function $f: \mathbb{R} \longrightarrow \mathbb{R}$ is smooth,

$$
f(s)=\left\{\begin{array}{cc}
\mathrm{e}^{-1 / s}, & \text { if } s>0 \\
0, & \text { if } s \leq 0
\end{array}\right.
$$

From it, it is possible to construct $\tilde{f}(s)=\frac{f(s)}{f(s)+f(1-s)}$ and $\bar{f}(s)=\tilde{f}(s+2) \tilde{f}(2-s)$, defined on $\mathbb{R}$, both non-negative and smooth. But $\bar{f}$ satisfies that if $s \in[-1,1]$, then $\bar{f}(s)=1$, and if $s \leq-2$ or $s \geq 2$, then $\bar{f}(s)=0$. By simple changes of parameters, we obtain the following lemma.

Lemma 5.1 Let $p_{1}, p_{2}, q_{1}$ and $q_{2} \in \mathbb{R} \cup\{-\infty,+\infty\}$ such that $-\infty \leq p_{1}<q_{1}<p_{2}<q_{2} \leq$ $+\infty$. Let $d=\min \left\{q_{1}-p_{1}, p_{2}-q_{1}, q_{2}-p_{2}\right\} / 4$. Then, there exist three smooth functions $\left.f_{i}:\right] p_{1}, q_{2}[\longrightarrow \mathbb{R}, i=0,1,2$, satisfying the following properties.

1. $0 \leq f_{i} \leq 1$, for $i=0,1,2$.

2. If $\left.s \in] p_{1}, q_{1}-d\right], f_{1}(s)=1$, and $f_{1}(s)=0$ if $s \geq q_{1}$.

3. If $s \in\left[q_{1}, p_{2}\right], f_{0}(s)=1$, and $f_{0}(s)=0$ if $s \geq p_{2}+d$ or $s \leq q_{1}-d$.

4. If $s \in\left[p_{2}+d, q_{2}\left[, f_{2}(s)=1\right.\right.$ and $f_{2}(s)=0$ if $s \leq p_{2}$.

Next, we would like to construct a new partly marginally trapped surface from two marginally trapped surfaces. We describe the method in several steps. 
Preliminaries. Given two unit curves, $\left.\alpha^{i}:\right] p_{i}, q_{i}\left[\longrightarrow \mathcal{P}, i=1,2\right.$, such that $\Sigma_{\alpha^{i}}$ is a marginally trapped surface, with $-\infty \leq p_{1}<q_{1}<p_{2}<q_{2} \leq+\infty$. Denote the associated functions by $\rho_{i}, \xi_{i}$ and $\varepsilon_{i}, i=1,2$. We write $\alpha^{i}=\left(\alpha_{1}^{i}, 0, \alpha_{3}^{i}, \alpha_{4}^{i}\right), i=1,2$ for the profile curves and define the numbers $m_{1}=\left(p_{2}+q_{1}\right) / 2$ and $m_{2}=\left(p_{2}+2 d-q_{1}\right) / 2$. Using Corollary 4.1, we consider the curve $\left.\alpha^{0}:\right] q_{1}-d, p_{2}+d[\longrightarrow \mathcal{P}$ which is the profile curve of an extremal surface $\Sigma_{\alpha^{0}}$, with $f(s)=\sqrt{m_{2}^{2}-\left(s+m_{1}\right)^{2}}$ and $\xi_{0}=0$.

Step 1. Using Lemma 5.1, we define the function $\left.\beta_{1}:\right] p_{1}, q_{2}\left[\longrightarrow \mathbb{R}\right.$ as $\beta_{1}=\sum_{i=0}^{2} f_{i} \alpha_{1}^{i}$, with the functions $f_{i}$ from Lemma 5.1. Clearly, this function is smooth and positive. Moreover, $\beta_{1}$ restricted to $] p_{1}, q_{1}-d\left[\right.$, ] $q_{1}, p_{2}[$ and $] p_{2}+d, q_{2}[$, respectively, is equal to $\alpha_{1}^{1}, \alpha_{1}^{0}$ and $\alpha_{1}^{2}$, restricted to the respective intervals.

Step 2. Define the smooth function $\rho$ as in Theorem 4.1 for $\beta_{1}$. Also, if $\left.s \in\right] p_{1}, q_{1}-d[$, then $\rho(s)=\rho_{1}(s)$; if $\left.s \in\right] q_{1}, p_{2}[$, then $\rho(s)=0$; and if $s \in] p_{2}+d, q_{2}\left[\right.$, then $\rho(s)=\rho_{2}(s)$.

Step 3. Define the function $\varepsilon:] p_{1}, q_{2}[\longrightarrow \mathbb{R}$, given by

$$
\varepsilon(s)=\left\{\begin{array}{ccc}
\varepsilon_{1} & \text { if } & \left.s \in] p_{1}, q_{1}\right], \\
0 & \text { if } & \left.s \in] q_{1}, p_{2}\right], \\
\varepsilon_{2} & \text { if } & s \in] p_{2}, q_{2}[.
\end{array}\right.
$$

It is a simple matter to check that the function $\varepsilon \rho$ is smooth.

Step 4. Define $\xi=\int \frac{\varepsilon \rho}{1+\left(\beta_{1}^{\prime}\right)^{2}} \mathrm{~d} s$, and $\beta_{3}, \beta_{4}$ as in Theorem 4.1. Thus, the unit curve $\beta=$ $\left(\beta_{1}, 0, \beta_{3}, \beta_{4}\right)$ is the profile curve of a partly marginally trapped surface.

Step 5. It only remains to show how the original surfaces are related with the new one. For this we resort to item 3 of Theorem 4.1. Thus, if $s \in] p_{1}, q_{1}-d\left[\right.$, then $\beta_{1}(s)=\alpha_{1}^{1}(s)$ and $\varepsilon(s)=\varepsilon_{1}$, and therefore, there exists a direct affine isometry $F_{1}: \mathbb{L}^{4} \longrightarrow \mathbb{L}^{4}$ such that

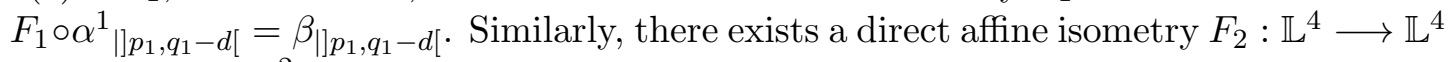

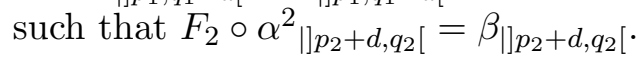

This method can be easily extended to a countable family of G-invariant partly marginally trapped surfaces, as it is shown in the following corollary.

Corollary 5.1 Let $\left\{\alpha^{k}:\right] a_{k}, b_{k}[\longrightarrow \mathcal{P} / k \in \mathcal{K} \subset \mathbb{N}\}$ be a countable family of unit curves with $a_{k}, b_{k} \in \mathbb{R}$ for any $k \in \mathcal{K}$, such that each $\Sigma_{\alpha^{k}}, k \in \mathcal{K}$, is a $\mathbf{G}$-invariant partly marginally trapped surface. Then, there exists a $\mathbf{G}$-invariant partly marginally trapped surface $\Sigma_{\alpha}$ such that for each $k \in \mathcal{K}$, there exists an open subset $C_{k}$ of the domain of $\alpha$, and an affine isometry $F_{k}$ of $\mathbb{L}^{4}$ satisfying $F_{k}\left(\Sigma_{\alpha^{k}}\right)=\Sigma_{\left.\alpha\right|_{C_{k}}}$.

\section{The Gaussian Curvature of G-Invariant Partly Marginally Trapped Surfaces}

Corollary 6.1 Let $\kappa: I \subset \mathbb{R} \longrightarrow \mathbb{R}$ be a smooth function. Given $s_{0} \in I$, there exist $\delta>0$ and a unit space-like curve $\alpha:\left(s_{0}-\delta, s_{0}+\delta\right) \longrightarrow \mathcal{P}$ such that $\alpha$ is a unit profile curve of the $\mathbf{G}$-invariant surface $\Sigma_{\alpha}$ whose Gaussian curvature satisfies $K(s, \theta)=\kappa(s)$ for any $(s, \theta) \in\left(s_{0}-\delta, s_{0}+\delta\right) \times \mathbb{R}$. In addition, 
1. The surface $\Sigma_{\alpha}$ is marginally trapped on the points of $\left(s_{0}-\delta, s_{0}+\delta\right)$ where $1+\left(\alpha_{1}^{\prime}\right)^{2}-$ $\kappa \alpha_{1}^{2} \neq 0$. Moreover, the mean curvature vector $H$ of the surface $\Sigma_{\alpha}$ is future-pointing if, and only if, $\kappa<\frac{1+\left(\alpha_{1}^{\prime}\right)^{2}}{\alpha_{1}^{2}}$.

2. The surface $\Sigma_{\alpha}$ is extremal on the points of $\left(s_{0}-\delta, s_{0}+\delta\right)$ where $1+\left(\alpha_{1}^{\prime}\right)^{2}-\kappa \alpha_{1}^{2}=0$.

Proof: Given a smooth function $\kappa: I \subset \mathbb{R} \longrightarrow \mathbb{R}$, let $\alpha_{1}:\left(s_{0}-\delta, s_{0}+\delta\right) \longrightarrow \mathbb{R}$ be a positive solution of the differential equation $\alpha_{1}^{\prime \prime}=-\kappa \alpha_{1}$ (recall expression (12)). Now, we only need to resort to Theorem 4.1, taking the constant $\varepsilon=1$. The condition $\rho<0$ becomes now $0>\rho=-\left(1+\left(\alpha_{1}^{\prime}\right)^{2}+\alpha_{1} \alpha_{1}^{\prime \prime}\right) / \alpha_{1}$. From here, it immediately follows that $\kappa<\frac{1+\left(\alpha_{1}^{\prime}\right)^{2}}{\alpha_{1}^{2}}$.

Corollary 6.2 There are no $\mathbf{G}$-invariant extremal surfaces with constant Gaussian curvature in $\mathbb{L}^{4}$.

Proof: Let $M$ be an extremal G-invariant surface with constant Gaussian curvature in $\mathbb{L}^{4}$. Then, there exists a unit curve $\alpha$ such that an open subset of $M$ is $\Sigma_{\alpha}$. According to Corollary 6.1, equation $\left(\alpha_{1}^{\prime}\right)^{2}=K \alpha_{1}^{2}-1$ holds on an open interval. This implies $K>0$. Next, by (12), there holds that $\alpha_{1}(s)=a_{1} \cos \left(\sqrt{K} s+a_{2}\right)$, for suitable integration constants $a_{1}, a_{2} \in \mathbb{R}$. However, for this $\alpha_{1}$, equation $\left(\alpha_{1}^{\prime}\right)^{2}=K \alpha_{1}^{2}-1$ holds only for some isolated points, which is a contradiction.

Corollary 6.3 A G-invariant marginally trapped surface $\Sigma_{\alpha}$ is flat if, and only if, its unit profile curve is, up to translations and orientation, $\alpha(s)=\left(\alpha_{1}(s), 0, \alpha_{3}(s), \alpha_{4}(s)\right.$, where

$$
\begin{aligned}
& \alpha_{1}(s)=a_{1} s+a_{2}>0, \\
& \alpha_{3}(s)=\frac{a_{1} s+a_{2}}{\sqrt{a_{1}^{2}+1}}\left(\varepsilon \sin \left(\varepsilon \frac{\log \left(a_{1} s+a_{2}\right)}{b_{1}}+\xi_{0}\right)+a_{1} \cos \left(\varepsilon \frac{\log \left(a_{1} s+a_{2}\right)}{b_{1}}+\xi_{0}\right)\right) \\
& \alpha_{4}(s)=\frac{a_{1} s+a_{2}}{\sqrt{a_{1}^{2}+1}}\left(\varepsilon \cos \left(\varepsilon \frac{\log \left(a_{1} s+a_{2}\right)}{b_{1}}+\xi_{0}\right)-a_{1} \sin \left(\varepsilon \frac{\log \left(a_{1} s+a_{2}\right)}{b_{1}}+\xi_{0}\right)\right),
\end{aligned}
$$

$a_{1}, a_{2}, \xi_{0} \in \mathbb{R}$ being integration constants, $\varepsilon= \pm 1$ and

$$
H=-\frac{\sqrt{1+a_{1}^{2}}}{a_{1} s+a_{2}}\left(-\eta_{1}+\varepsilon \eta_{2}\right) .
$$

Proof: It is sufficient to recall that the surface is flat if, and only if, $K=0$, and by (12), it follows that the function $\alpha_{1}$ can be expressed as $\alpha_{1}(s)=a_{1} s+a_{2}$ for some integration constants $a_{1}, a_{2} \in \mathbb{R}$. Now, we only have to resort to Theorem 4.1.

\section{Examples}

Example 7.1 We consider the unit space-like curve

$$
\alpha: \mathbb{R} \longrightarrow \mathbb{L}^{4}, \alpha(s)=\left(1+\frac{s^{2}}{4}, 0,-3 s+8 \arctan (s / 2), 4 \log \left(1+\frac{s^{2}}{4}\right)-\frac{s^{2}}{4}\right) .
$$

Let $\Sigma_{\alpha}$ be the G-invariant surface whose profile curve is $\alpha$. Then, this surface is pure, future-pointing, marginally trapped, with

$$
H=\frac{3}{\sqrt{2} \sqrt{4+s^{2}}} \mathbf{l}, \quad K=\frac{-2}{4+s^{2}} .
$$


This is done by putting $\rho=-3 / 2, c=1$ and $\varepsilon=-1$ in Theorem 4.1, and letting all integration constants be zero.

Example 7.2 Given $c \in \mathbb{R}, c>0$, the spacelike curve $\alpha: \mathbb{R} \longrightarrow \mathbb{L}^{4}$, given by

$$
\alpha(s)=(c \cosh (s), 0,4 c \arctan (\tanh (s / 2))-c \sinh (s), 2 c \log (\cosh (s))), \quad g_{o}\left(\alpha^{\prime}, \alpha^{\prime}\right)=c^{2},
$$

is a profile curve of a G-invariant, future-pointing, marginally trapped surface $\Sigma_{\alpha}$ with constant Gaussian curvature $K=-1 / c^{2}$ and $H=\frac{-2 \sqrt{2}}{c}$ l. In fact, this example has been computed by choosing $\alpha_{1}(s)=c \cosh (s), c>0, \varepsilon=-1$ and making use of Theorem 4.1.

Example 7.3 We consider the unit curve $\alpha:] 0, \pi[\longrightarrow \mathcal{P}$ given by

$$
\alpha_{1}(s)=\sin (s), \alpha_{3}(s)=\int_{s} \sqrt{1+\cos ^{2}(u)} \cos (\xi(u)) \mathrm{d} u, \alpha_{3}(s)=\int_{s} \sqrt{1+\cos ^{2}(u)} \sin (\xi(u)) \mathrm{d} u,
$$

where $\xi(u)=\arctan (\cos (u))+\log (\tan (u / 2))$. According to Theorem 4.1, the G-invariant surface generated by $\alpha$ is partly marginally trapped. Indeed, $\rho(s)=-\frac{2 \cos ^{2}(s)}{\sin (s)} \leq 0$ and the constant $\varepsilon$ takes the value $\varepsilon=-1$. This surface is not (pure) marginally trapped because $\rho(\pi / 2)=0$. Moreover, by (12), we see

$$
K=1, \quad H=\frac{\sqrt{2} \cos ^{2}(s)}{\sin (s) \sqrt{1+\cos ^{2}(s)}} \mathbf{l} .
$$

Example 7.4 In this example, we use the procedure outlined in Section 5 to construct a unit profile curve of a partly marginally trapped surface such that the mean curvature vector is null, future-pointing on one region, zero on a second region with non-empty interior and null, future-pointing on a third region. In [9] these surfaces are called null future-trapped and denoted by $\nwarrow$. $\nearrow$. For the sake of simplicity, we let all the integration constants be zero.

First, we consider two unit curves $\alpha, \beta: \mathbb{R} \longrightarrow \mathcal{P}$,

$$
\begin{aligned}
& \alpha(s)=\left(s^{2}+1,0,-s \sqrt{s^{2}+1}+2 \operatorname{arcsinh}(s),-3 \sqrt{s^{2}+1}\right), \\
& \beta(s)=\left(s^{2}+1,0,-s \sqrt{s^{2}+1}+2 \operatorname{arcsinh}(s), 3 \sqrt{s^{2}+1}\right) .
\end{aligned}
$$

Next, we choose a unit curve that generates an extremal surface, $\varphi:]-2,2[\longrightarrow \mathcal{P}$,

$$
\varphi(s)=\left(\sqrt{4-s^{2}}, 0,2 \arcsin \left(\frac{s}{2}\right), 0\right) .
$$

A straightforward computation shows that, according to Theorem 4.1, the associated $\rho$ functions are

$$
\rho_{\alpha}(s)=-\frac{6 s^{2}+3}{s^{2}+1}=\rho_{\beta}(s), \quad \rho_{\varphi}(s)=0 .
$$

Since $\rho_{\alpha}$ and $\rho_{\beta}$ are negative, both surfaces $\Sigma_{\alpha}$ and $\Sigma_{\beta}$ are future-pointing. Further, the associated $\varepsilon$ functions are taken to be the constants $\varepsilon_{\alpha}=1$ and $\varepsilon_{\beta}=-1$. In order to recall the procedure of Section 5, we restric the curves to

$$
\alpha:]-\infty,-1[\longrightarrow \mathcal{P}, \quad \beta:] 1,+\infty[\longrightarrow \mathcal{P} .
$$


Example 7.5 A $*$-surface can be constructed by choosing the unit curves $\alpha:] 3,+\infty[\rightarrow \mathcal{P}$,

$$
\alpha(s)=\left(s^{2}+1,0,-s \sqrt{s^{2}+1}+2 \operatorname{arcsinh}(s),-3 \sqrt{s^{2}+1}\right),
$$

and $\beta:]-\frac{3}{2}, 2[\rightarrow \mathcal{P}$,

$$
\beta(s)=\left(\sqrt{-\frac{3}{2} s^{2}+2 s+2}, 0, \beta_{3}(s), \beta_{4}(s)\right)
$$

with

$$
\beta_{3}(s)=\int \sqrt{\frac{3 s^{2}-4 s+12}{-6 s^{2}+8 s+8}} \cos (\xi(s)) \mathrm{d} s, \quad \beta_{4}(s)=\int \sqrt{\frac{3 s^{2}-4 s+12}{-6 s^{2}+8 s+8}} \sin (\xi(s)) \mathrm{d} s,
$$

and

$$
\xi(s)=-\frac{\sqrt{6}}{3} \arcsin \left(\frac{3 s-2}{4}\right)+\arctan \left(\frac{-3 s+2}{\sqrt{-6 s^{2}+8 s+8}}\right) .
$$

Both curves are the profile curves of a future and past marginally trapped surface, respectively. Then, the procedure from Section 5 gives different surfaces according to the chosen values of $\varepsilon_{\alpha}$ and $\varepsilon_{\beta}$, i. e.,

\begin{tabular}{|c|c|c|c|}
\hline \multicolumn{2}{|c|}{ null untrapped } & \multicolumn{2}{|c|}{ null dual } \\
\hline$\nearrow$ & $\nwarrow$ & $\nwarrow$ & $\nearrow$ \\
$\searrow$ & $\swarrow$ & $\searrow$ & $\swarrow$ \\
$\varepsilon_{\alpha}=-1$ & $\varepsilon_{\alpha}=1$ & $\varepsilon_{\alpha}=1$ & $\varepsilon_{\alpha}=-1$ \\
$\varepsilon_{\beta}=-1$ & $\varepsilon_{\beta}=1$ & $\varepsilon_{\beta}=-1$ & $\varepsilon_{\beta}=1$ \\
\hline
\end{tabular}

Moreover, if we also consider $\gamma:]-\infty,-3[\longrightarrow \mathcal{P}, \gamma(s)$ with the same expression as $\alpha(s)$, by iterating the procedure, it is possible to obtain a surface of type

\section{Conclusions}

Among the partly marginally trapped surfaces in Minkowski 4-space, we are interested in the study of those which are invariant by boost isometries. As a main result, we are able to classify them in Theorem 4.1. From this, we obtain a fairly long list of corollaries. First, we obtain a classification of boost invariant extremal surfaces. Next, a careful reading of Theorem 4.1 gives rise to a method to construct partly marginally trapped surfaces in Minkowski 4-space which may include regions which are future or past-pointing, as desired, and extremal regions in between. As an application, we obtain examples of proper $*$-surfaces. Also, we show that it is possible to construct a boost invariant surface with prescribed Gaussian curvature. In particular, we show the non-existence of boost invariant, extremal surfaces with constant Gaussian curvature. In the text we make use of the notation introduced in [9].

These methods may lead to the study of boost invariant generalized horizons in Minkowski 4-space, since they are hypersurfaces foliated by marginally trapped surfaces. These techniques may also be applied to the study of marginally trapped surfaces which are invariant by other subgroups of isometries in the Minkowski 4-space, as well as in other space-times. 


\section{References}

[1] B.-Y. Chen and J. Van der Veken, Marginally trapped surfaces in Lorentzian space forms with positive relative nullity, Class. Quantum Grav. 24 (2007), 551-563.

[2] B.-Y. Chen and J. Van der Veken, Spatial and Lorentzian surfaces in Robertson-Walker space times, J. Math. Phys. 48 (2007), 073509.

[3] M. Dafermos, Spherically symmetric spacetimes with a trapped surface, Class. Quantum Grav. 22 (2005), 2221-2232.

[4] G. Ellis, Closed trapped surfaces in cosmology, Gen. Relativ. Grav. 35 (2003), 1309-1319.

[5] E. Malec and N. Ó Murchadha, Trapped surfaces in cosmological spacetimes, Phys. Rev. D 54 (1996), 4799-4804.

[6] M. Mars and J. Senovilla, Trapped surfaces and symmetries, Class. Quantum Grav. 20 (2003), L293-L300.

[7] R. Penrose, Gravitational collapse and space-time singularities, Phys. Rev. Lett. 14 (1965), 57-59.

[8] J. Senovilla, Trapped surfaces, horizons and exact solutions in higher dimensions, Class. Quantum Grav. 19 (2002), L113-L119.

[9] J. Senovilla, Classification of spacelike surfaces in spacetime, Class. Quantum Grav. 24 (2007), 3091-3124.

[10] D. Struik, Lectures on Classical Differential Geometry, second edition, Addison-Wesley Publishing Company, Reading Massachusetts, 1961.

[11] G. Vranceanu and R. Rosca, Introduction in relativity and pseudo-Riemannian geometry, Academiei Republicii Socialiste Romania, Bucarest, 1976.

Stefan Haesen

Department of Mathematics

Katholieke Universiteit Leuven

Celestijnenlaan 200B

3001 Leuven, Belgium

E-mail: Stefan.Haesen@wis.kuleuven.be

Miguel Ortega

Departamento de Geometría y Topología

Universidad de Granada

18071 Granada, Spain

E-mail: miortega@ugr.es 